\title{
Mid-brain pathology of Wilson's disease: MRI analysis of three cases
}

\author{
Seiji Hitoshi, Makoto Iwata, Kohki Yoshikawa
}

\begin{abstract}
MRI scans were obtained from three patients with Wilson's disease, all of whom showed rigidity and dysarthria; two also showed tremor and dystonia. Two had been treated with D-penicillamine for seven and 14 years, respectively and their neurological abnormalities had improved, but the third patient had not been treated. T2weighted MRI of the mid-brain in all three revealed the characteristic "face of the giant panda" sign, consisting of high signal intensity in the tegmentum except for red nucleus, preservation of signal intensity of the lateral portion of the pars reticulata of the substantia nigra and hypo-intensity of the superior colliculus. The clinical significance of these MRI abnormalities is discussed.
\end{abstract}

MRI provides more detailed anatomical information than CT of the brain especially of the structure of the basal ganglia and brain-stem. It also provides biochemical information on the distribution of heavy metal in the brain substance; MRI is clearly a useful method for assessing heavy metal storage disease. In Wilson's disease, emphasis has hitherto been laid on lesions of the lenticular nucleus and thalamus, and atrophy of the cerebral and cerebellar cortex. In this paper three cases of Wilson's disease are reported and their MRI analysed, particularly abnormalities of the brain stem.

\section{Case reports}

Case 1 was a 39 year old male who had developed sialorrhoea and dysarthria at the age of 17 years, and showed severe intention tremor in the upper extremities by the age of 21. The diagnosis of Wilson's disease was made when he was 25 , and he had since been treated with D-penicillamine. His symptoms had improved and he exhibited only minimal rigidity and dysarthria.

Case 2 was a 23 year old female who had first noticed finger tremor and dysarthria when she was 11 years old. A diagnosis of Wilson's disease was made at the age of 16 , and she had been treated with D-penicillamine. On examination a resting tremor was found in the left upper and both lower extremities, and rigidity, dysarthria and a mild dystonic posture were also seen.

Case 3 was a 17 year old male who first exhibited sialorrhoea and dysarthria when he was nine years old. Neurological examination at the age of 17 revealed dysarthria, finger tremor, rigidity and a dystonic posture, and the diagnosis of Wilson's disease was made. $\mathrm{He}$ had received no treatment.

A 40 year old male.and a 21 year old female, both free from neurological abnormalities, were used as controls for MRI brain scanning.

\section{Method and results}

Images were obtained with a Siemens Magnetom scanner with a superconducting magnet of 1.5 Tesla field strength, spin echo $\mathrm{T} 1$-weighted $(\mathrm{TR}=500 \mathrm{~ms}, \mathrm{TE}=17 \mathrm{~ms})$ and $\mathrm{T} 2$-weighted ( $\mathrm{TR}=3000 \mathrm{~ms}, \mathrm{TE}=$ $90 \mathrm{~ms}$ ). Proton density images were also obtained by the long spin echo method ( $\mathrm{TR}=3000 \mathrm{~ms}, \mathrm{TE}=22 \mathrm{~ms}$ ).

Atrophy of the mid-brain and pontine tegmentum was present in all three patients. The cerebral and cerebellar cortex and the medulla oblongata were also atrophic.

In the three patients the T2-weighted images revealed several abnormalities of signal intensity. In normal subjects, the putamen presents a signal intensity slightly higher than the cerebral cortex, and the globus pallidus shows a marked hypo-intensity. In cases 1 and 2 , marked hypo-intensity was observed in the posterolateral part of the putamen, while areas of spotty hyper-intensity were seen in the rest of the putamen. Diffuse hyper-intensity of the putamen was found in case 3, without any area of hypo-intensity. A hyper-intense area was observed in the globus pallidus in cases 1 and 2 (fig 1).

The superior colliculus, which in normal subjects shows the same signal intensity as cerebral white matter, showed a remarkable decrease in signal intensity in the patients with Wilson's disease. A reversal of signal intensity ${ }^{1}$ was seen in the lateral portion of the substantia nigra, that is, the marked hypointensity chracteristic of the normal substantia nigra, was lost in cases 1 and 2 . In these patients, it showed almost the same signal level as cerebral white matter; case 3 showed a normal hypo-intensity in that area. The thalamus and the tegmentum of the mid-brain showed a marked hyper-intensity, especially around the red nucleus, which retained their normal hypo-intensity. As a result, the T2weighted horizontal section of the mid-brain in these patients showed the "face of giant panda" appearance, which we describe for the first time and is specific for Wilson's disease (fig 2). Hyper-intensity in the T2-weighted image was also observed in the pontine tegmentum, medulla oblongata, and the cerebellar white matter around the dentate nucleus; the latter structures were thought to be normal (fig 3). 
Figure 1 Axial T2weighted MRI made at the level of the basal ganglia in four subjects reveal

abnormal signal intensities in the putamen $(P)$, globus pallidus (GP), and thalamus $(T)$ in three cases of Wilson's disease. $\mathrm{NC}=$ normal control, $a$ = case $1, b=$ case $2, c=$ case 3.
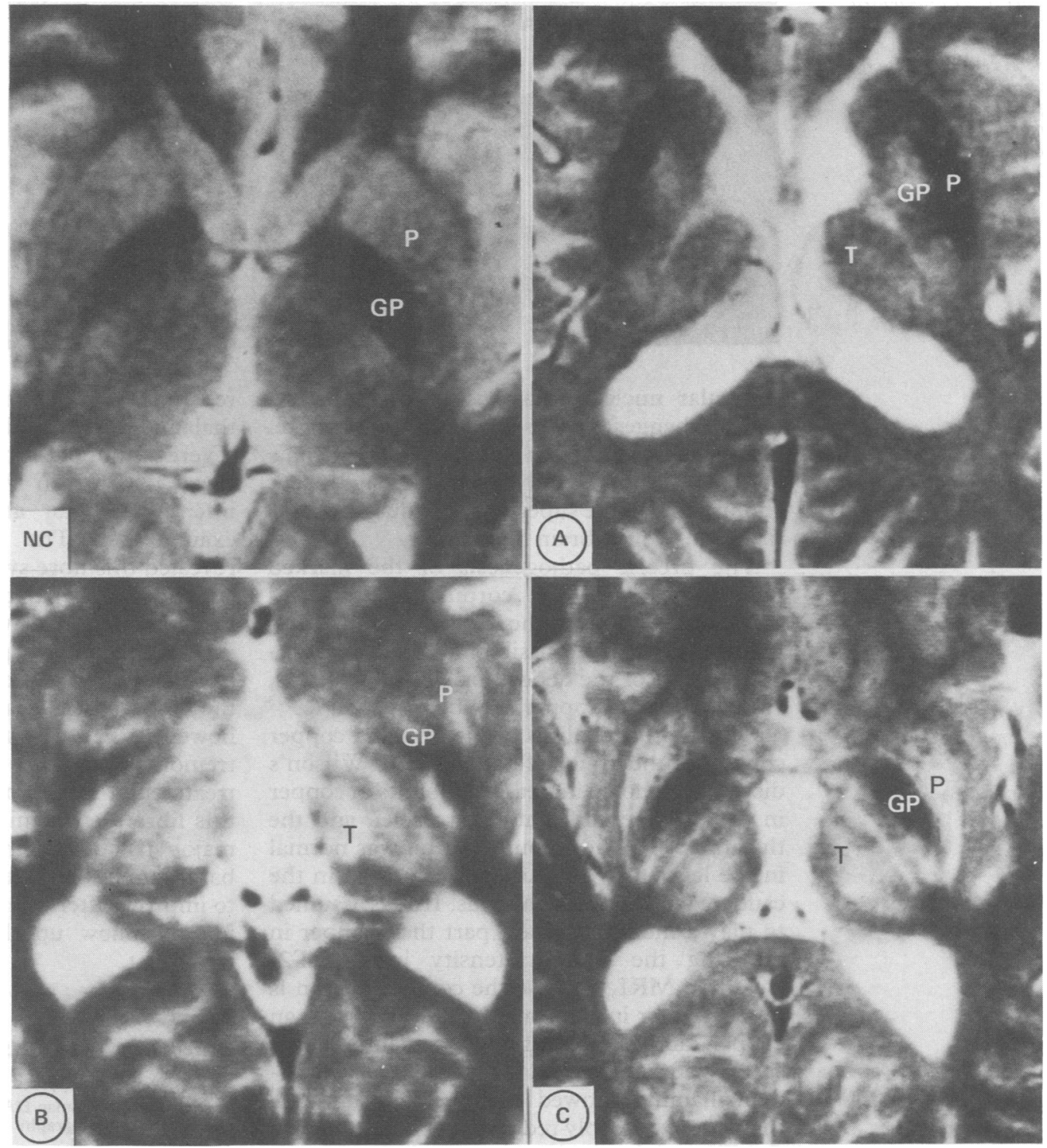

\section{Discussion}

Several descriptions have been given of the abnormalities found in Wilson's disease, as shown by $\mathrm{CT}^{2-5}$ and MRI. ${ }^{6-10}$ Atrophy of the cortex of the cerebral and cerebellar hemispheres and the brain-stem has also been described, as well as abnormalities of the lenticular and caudate nuclei. MRI studies reported hyper-intensity in T2-weighted images of the thalamus, mid-brain, pons, and dentate nucleus. Most of these studies used MRI systems with a magnetic field lower than 0.5 Tesla. ${ }^{6-8}$ Alternatively, studies made with a high field MRI system (1.5 Tesla) have shown hypo-intense basal ganglia in T2-weighted images ${ }^{1910}$; so far there have been no reports on the superior colliculus, substantia nigra, and mid-brain tegmentum in Wilson's disease.

Microscopic examinations of the brain in cases of Wilson's disease have revealed neuronal loss, spongiform degeneration, gliosis, or cavitation in the basal ganglia, brain stem, and the cerebral and cerebellar white matter. ${ }^{11-12}$ Increased signal intensity in the T2-weighted MRI, which is seen in the cerebral and cerebellar white matter and the
Figure 2 Axial T2-weighted MRI of the mid-brain in four subjects reveal the "face of giant panda" appearance in three cases of Wilson's disease. NC = normal control, $a=$ case $1, b=$ case $2, c=$ case $3, S N=$ substantia nigra, $S C=$ superior colliculus, $R=$ red nucleus. 
Figure 3 T2-weighted images at the levels of pons (a) and cerebellum (b) in case 2 , reveal hyperintensity in the pontine tegmentum and the cerebellar white matter around dentate nucleus (D).
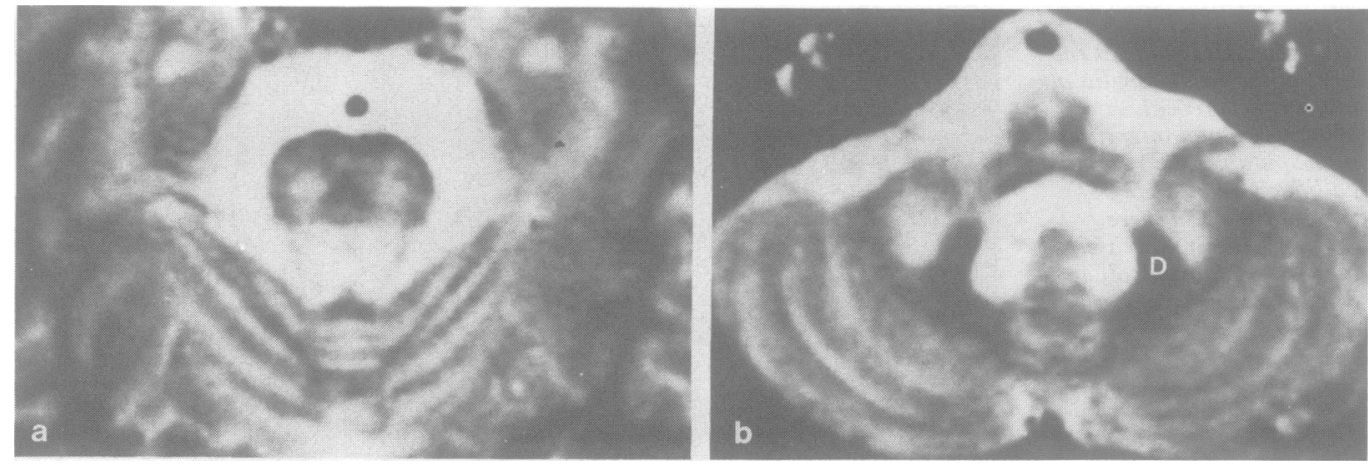

lenticular nucleus, may reflect these pathological changes, or the oedema they provoke. Necropsy examination in cases of Wilson's disease does not usually reveal any significant abnormality of the superior colliculus, substantia nigra, or mid-brain tegmentum.

The precise pathogenesis of the marked hypo-intensity of the corpus striatum and superior colliculus in the T2-weighted MRI is unknown, but it is possible that the paramagnetic effects of the deposition of heavy metals, such as iron and copper may be responsible. ${ }^{13}$

Quantitative analysis of the iron and copper content of the brains of patients with Wilson's disease has shown increased amounts of copper in the caudate and lenticular nuclei, and the thalamus. ${ }^{14-16}$ The amount of iron was normal in the lenticular nucleus, but increased in the caudate nucleus and thalamus. Iron is assumed to play a more important part than copper in reducing the signal intensity in the $T 2$ weighted MRI, because the content of iron is much greater in cases of Wilson's disease than copper, and because the two patients in this series who were treated for many years with $\mathrm{D}$ penicillamine showed identical appearances, that is, considerably reduced signal intensity in the T2-weighted MR images, similar to the untreated patient. To date no reports have compared heavy metal amounts in the substantia nigra or superior colliculus between normal subjects and cases with Wilson's disease. It will be necessary to correlate the changes in signal intensity in MRI imaging with the heavy metal content of each structure in the basal ganglia and brain stem.

A recovery in signal intensity in the substantia nigra in cases of Parkinson's disease and the Parkinson-plus syndrome was first reported by Rutledge et al. ${ }^{1}$ Although it is difficult to correlate neurological findings with abnormalities on MRI, cases 1 and 2 showed a recovery of signal intensity in the lateral part of the substantia nigra and presented clinically with rigidity, which can be attributed to a lesion of the substantia nigra. Case 2 exhibited a resting tremor, another characteristic feature of the Parkinsonian syndrome. Case 3 also presented with rigidity, but showed the more usual finding, hypo-intensity in the lateral part of the substantia nigra in the T2-weighted images. It is possible that the duration of the disease process may contribute to the MRI changes, or that treatment with D-penicillamine may be responsible for them.

The mid-brain tegmentum, especially the red nucleus, contains the cerebello-rubrothalamic tract, and damage to this tract causes severe tremor of the intention type. None of the three patients in this series exhibited any intention tremor at the time of the MRI examination. The MRI of these patients revealed this fibre system to be intact, and the superior cerebellar peduncle and its decussaT1- and the T2-weighted images, and normal hypo-intensity of the dentate and red nuclei in the T2-weighted images was also noted. Case 1 however, had shown very severe intention tremor before the start of D-penicillamine treatment 14 years previously. ${ }^{17}$ The lesion of this fibre system might be reversible without major structural damage. Abnormalities in the basal ganglia on CT examination were reported to improve after treatment. ${ }^{4}$ It will be interesting to follow up the MRI of case 3 after treatment.

1 Rutledge JN, Hilal SK, Silver AJ, et al. Study of movement disorders and brain iron by MR. Am J Roentogenol 1987;149:365-79.

2 Nelson RF, Guzman DA, Grahovac Z, et al. Computerized cranial tomography in Wilson disease. Neurology 1979;29:866-8.

3 Ropper AH, Hatten HP, Davis KR. Computed tomography in Wilson disease: report of 2 cases. Ann Neurol in Wilson

4 Williams FJB, Walshe JM. Wilson's disease: an analysis of the cranial computerized tomograpic appearances found in 60 patients and the changes in response to treatmen with chelating agents. Brain 1981;104:735-52.

5 Takano K, Kuroiwa Y, Shimada Y, et al. CT manifestation of cerebral white matter lesion in Wilson disease. Ann Neurology 1983;13:108-9.

6 Lawler GA, Pennock JM, Steiner RE, et al. Nuclear magnetic resonance (NMR) imaging in Wilson disease. J Comp Ass Tomog 1983;7:1-8.

7 Aisen AM, Martel W, Gabrielsen TO, et al. Wilson disease of the brain: MR imaging. Radiology 1985;157:137-41.

8 Starosta-Rubinstein S, Young AB, Kluin K, et al. Clinical assessment of 31 patients with Wilson's disease. Correlations with structural changes on magnetic resonance imaging. Arch Neurology 1987;44:365-70.

9 Tsuchiya K, Makita K, Furui S, et al. Unusual MR signals (prolonged T1 and shortened and partially prolonged T2) in the basal ganglia in Wilson's disease. Journal of Medical in the basal ganglia in Wil.

10 Uchino A, Miyoshi T, Maeoka N, et al. MR imaging of the brain in Wilson's disease. Japanese Journal of Clinical Radiology 1989;34:1413-16.

11 Wilson SAK. Progressive lenticular degeneration: a familia nervous disease associated with cirrhosis of the liver. Brain 1912;34:295-509.

12 Greenfield JG. Familial hepatolenticular degeneration (Wilson's disease). In: Corsellis JAN, Adams JH, eds. Neuropathology. London: Arnold, 1984:595-9.

13 Drayer B, Burger P, Darwin R, et al. Magnetic resonance imaging of brain iron. Am J Neuroradiol 1986;7:373-80.

14 Cumings JN. The copper and iron content of brain and liver in the normal and in the hepato-lenticular degeneration. Brain 1948;71:410-15.

15 Warren PJ, Earl CJ, Thompson RHS. The distribution of copper in human brain. Brain 1961;83:709-17.

16 Cumings JN. Trace metals in the brain and in Wilson's disease. J Clin Path 1968;21:1-7.

17 Iwata M. Pathodynamics of tremor. Shinkei Kenkyu no Shinpo (Tokyo) 1985;29:210-21. tion to be relatively well preserved in both the 